Journal for ImmunoTherapy of Cancer

\title{
Impact of tumor mutational burden on checkpoint inhibitor drug eligibility and outcomes across racial groups
}

\author{
David Hsiehchen, ${ }^{1,2}$ Magdalena Espinoza, ${ }^{3}$ Cristina Valero, ${ }^{4}$ Chul Ahn, ${ }^{5}$ \\ Luc G T Morris ${ }^{4}$
}

To cite: Hsiehchen D, Espinoza M, Valero C, et al. Impact of tumor mutational burden on checkpoint inhibitor drug eligibility and outcomes across racial groups. Journal for ImmunoTherapy of Cancer 2021;9:e003683. doi:10.1136/ jitc-2021-003683

- Additional supplemental material is published online only. To view, please visit the journal online (http://dx.doi.org/10. 1136/jitc-2021-003683).

Accepted 01 November 2021

Check for updates

(c) Author(s) (or their employer(s)) 2021. Re-use permitted under CC BY-NC. No commercial re-use. See rights and permissions. Published by BMJ.

For numbered affiliations see end of article.

Correspondence to Dr David Hsiehchen; gbtwnow@gmail.com

\section{ABSTRACT}

The FDA approval of immune checkpoint inhibitors for cancers with tumor mutation burden (TMB) of at least 10 mut/Mb is postulated to reduce healthcare disparities by broadly expanding treatment eligibility. In a cohort of 39,400 patients with available genomic and race data, black and Asian patients were less likely to have TMB-high cancers in multiple types of malignancies based on the currently approved cut-off. Decreasing TMB thresholds preferentially increased the eligibility of minority patients for immune checkpoint inhibitors while retaining predictive value of treatment benefit in a cohort of immune checkpoint inhibitor treated patients. This study highlights differing distributions of TMB-high cancers between racial groups and provides guidance in developing more rational eligibility criteria for immune checkpoint inhibitors.

\section{INTRODUCTION}

Given the transformative impact of immune checkpoint inhibitors (ICIs) on cancer outcomes for a subset of patients, biomarkers to guide selection of patients who benefit from immunotherapies have been intensely investigated. Early observations in melanoma and non-small cell lung cancer demonstrated that ICI activity was associated with tumor mutation burden (TMB), which ultimately contributes to neoantigen load. ${ }^{2}$ TMB may explain differences in ICI response rates across different types of cancers, with malignancies associated with high rates of mutagenesis such as skin and lung cancers being among the most ICI sensitive cancers. ${ }^{3}$ Multiple lines of evidence support an association between TMB and ICI activity within multiple cancer types, though no consensus has been reached in determining how TMB should be applied as a predictive biomarker. ${ }^{4}$

Based on the phase 2 KEYNOTE-158 trial, pembrolizumab was approved by the Food and Drug Administration (FDA) for patients with refractory solid cancers harboring a TMB $\geq 10$ mut $/ \mathrm{Mb}^{5}$ Such cancers with a $\mathrm{TMB} \geq 10$ mut $/ \mathrm{Mb}$ were labeled as TMB-high cancers and associated with greater response rates. ${ }^{6}$ However, TMB-high cancers were not shown to be associated with longer median progression-free or overall survival. ${ }^{6}$ In addition, cancers with $<10$ mut/MB had nontrivial response rates and lower TMB cutoffs were not precisely explored, bringing into question the clinical and scientific basis of the FDA approved cut-off. ${ }^{6}$ While the expanded eligibility of patients to immunotherapies is purported to reduce cancer treatment disparities, racial differences in TMB and ICI treatment outcomes have not been investigated. ${ }^{7}$ Here, we demonstrate that cancers in Black and Asian patients were less likely to harbor TMB $\geq 10$ mut/Mb across multiple cancer types. Thus, the 10 mut/Mb cut-off may result in fewer non-White patients meeting eligibility for ICIs, although this imbalance would be alleviated by lowering the definition of high TMB. In a large cohort of ICI-treated patients, we show that lower numerical cutoffs for high TMB retain predictive value of ICI benefit regardless of race, which may have implications for determining ICI eligibility.

\section{TMB-high cancers are less frequent in blacks and Asians across two large genomic cohorts} We analyzed data from among the two largest tumor sequencing efforts to date including The Cancer Genome Atlas (TCGA) spanning 30 cancer types $(n=8347)$ and Memorial Sloan Kettering-Integrated Mutation Profiling of Actionable Cancer Targets (MSK-IMPACT) cohorts spanning 49 cancer types $(n=31,053)$. All eligible patients included adults with solid cancers and race data (see online supplemental methods). TMB was calculated as the number of all non-synonymous, somatic mutations identified per $\mathrm{Mb}$ of the length of genome sequenced. Using the $10 \mathrm{mut} / \mathrm{Mb}$ cut-off, TMB-high cancers (excluding melanomas where a majority of cases would have been classified as TMB-high) accounted for $10.3 \%$ and $11.3 \%$ of cases in TCGA and MSKIMPACT cohorts, respectively. Across both 


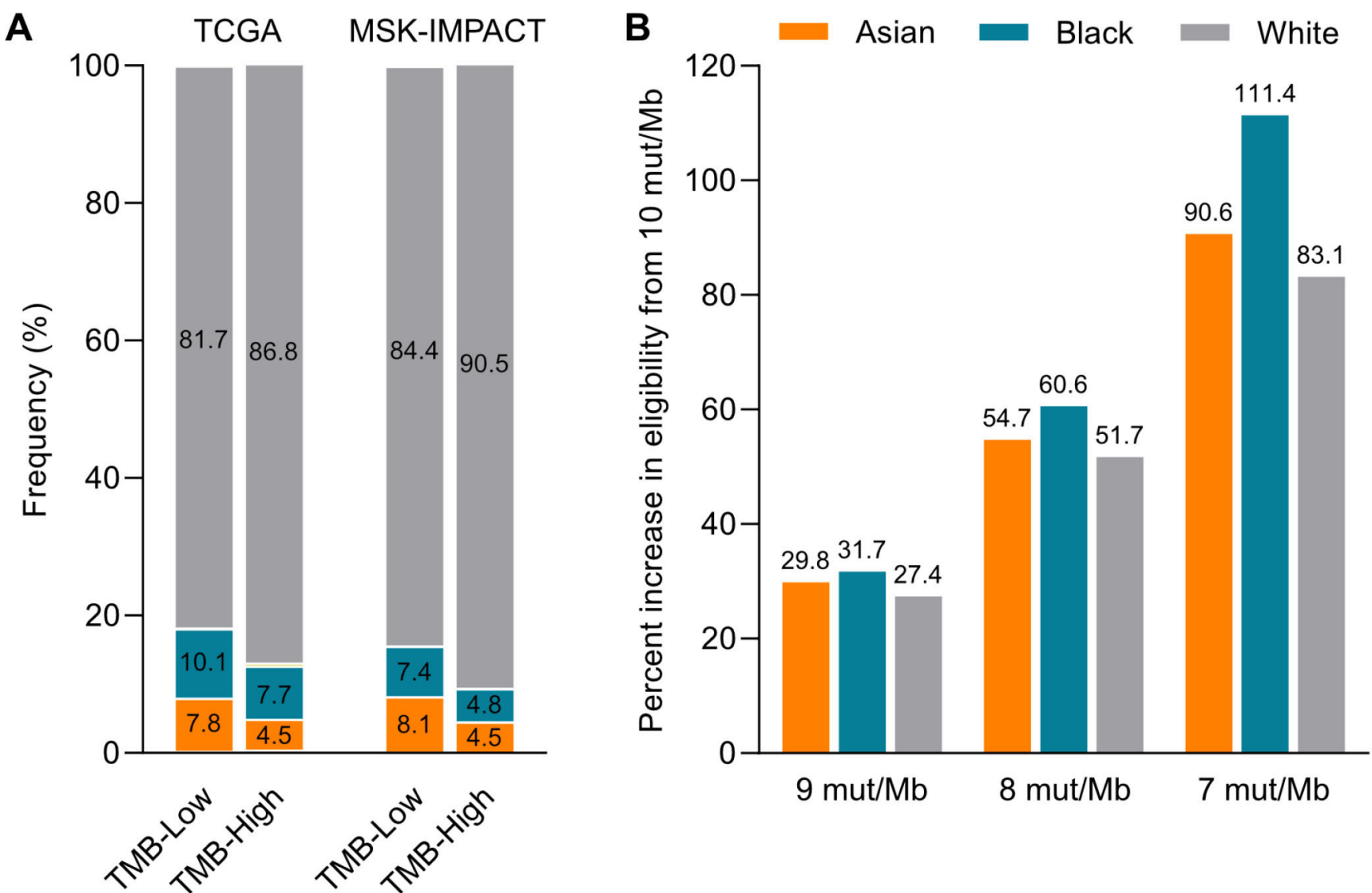

Figure 1 Race of eligible patients for immunotherapies and tumor mutation burden (TMB) cutoffs. (A) Proportions of patients with TMB-high or TMB-low cancers by race across The Cancer Genome Atlas (TCGA) and Memorial Sloan Kettering-Integrated Mutation Profiling of Actionable Cancer Targets (MSK-IMPACT) cohorts. In the TCGA cohort, blacks (OR 0.72; $95 \% \mathrm{Cl} 0.55$ to $0.93, p=0.01)$ and $A$ sian $(\mathrm{OR} 0.54 ; 95 \% \mathrm{Cl} 0.39$ to $0.76, \mathrm{p}<0.001)$ patients were less likely to have cancers with high TMB. Similarly, in the MSK-IMPACT cohort, blacks (OR, $0.61 ; 95 \% \mathrm{Cl} 0.52$ to $0.72, \mathrm{p}<0.001)$ and Asian $(\mathrm{OR}, 0.52 ; 95 \% \mathrm{Cl} 0.44$ to $0.62, p<0.001)$ patients were less likely to have cancers with high TMB. (B) Percent increase in patients across different race eligible for immunotherapy based on different TMB cutoffs.

cohorts, black (237/2787; OR 0.64; $95 \%$ CI 0.55 to 0.73 , $\mathrm{p}<0.001)$ and Asian $(198 / 2789$; OR $0.53 ; 95 \%$ CI 0.49 to $0.62, \mathrm{p}<0.001)$ patients were less likely to have cancers with high TMB, compared with white patients $(3925 / 29356)$ (figure 1A and online supplemental table 1). Genetic ancestry could be determined for the TCGA cohort, and categorizing patients by ancestry assignments generated from a prior TCGA Network analysis showed that patients of African (OR $0.77 ; 95 \%$ CI 0.60 to $0.98, \mathrm{p}=0.04$ ) and Asian (OR 0.62; 95\% CI 0.45 to $0.84, \mathrm{p}=0.002$ ) descent were less likely to have cancers with high TMB, compared with patients of European descent (online supplemental figure 1A). ${ }^{8}$ Even when restricting the analysis to tumor types enrolled in the KEYNOTE-158 trial with at least a $1 \%$ prevalence of TMB-high cancers, Black and Asian patients were less likely to have TMB-high cancers (online supplemental figure 1B). Decreasing the TMB cut-off preferentially increased the proportion of eligible black and Asian patients (figure 1B). The sparsity of patients of other races in either cohort precluded analyses of other racial minority groups.
Eligible patient demographics may be affected by differences in the proportion of constituent cancer types, particularly as some cancers may be more frequent in one racial group or associated with higher TMBs. However, logistic regression analysis showed that after adjusting for age, sex, cohort, stage, biopsy site (primary vs metastasis), and cancer type, black (OR $0.76 ; 95 \%$ CI 0.65 to $0.89, \mathrm{p}=0.001$ ) and Asian (OR $0.65 ; 95 \%$ CI 0.55 to $0.77)$ patients were less likely to have TMB-high cancers (online supplemental table 2). We repeated the regression analysis after patients were stratified by cancer type which showed a significant or trend association between TMB-high cancers and White patients in multiple cancer types including bladder, colon, breast, unknown primary, and endometrial cancers (online supplemental table 3). Exceptions were found in renal cell carcinomas where TMB-high cancers were enriched in black and Asian patients, and in non-small cell lung cancers where TMBhigh cancers were enriched in black patients but reduced in Asian patients. 
Neither the TCGA or MSK-IMPACT were designed as population-based studies. To examine the generalizability of the combined TCGA and MSK-IMPACT cohort, we assessed the correlation between the number of cases sequenced per cancer type and US cancer incidence based on Surveillance, Epidemiology, and End Results (online supplemental figure 2). We found a high correlation among 38 cancer types with available incidence data which accounted for $97 \%$ of all sequenced cancers $(\mathrm{p}<0.001)$. The correlation coefficient of 0.87 indicates that $75 \%$ of the variance in cases sequenced across different cancer types is explained by US cancer incidence.

\section{Outcomes among TMB-high and TMB-low cancers are treatment context dependent}

To assess whether a TMB cut-off of $10 \mathrm{mut} / \mathrm{Mb}$ is associated with clinical outcomes across patients of different race, we analyzed subsets of patients in the MSK-IMPACT cohort with available outcome data who were treated with systemic therapies for advanced cancers. In the non-ICI cohort, 7600 patients comprised of 6396 white, 553 black, and 651 Asian patients received non-ICI systemic agents. ${ }^{9}$ In the ICI cohort, 1867 patients including 1604 white, 128 black, and 135 Asian patients were treated with antiPD-1/PD-L1/CTLA-4 therapies as any line of therapy. ${ }^{9} \mathrm{We}$ used multivariable Cox regression analyses to determine if $\mathrm{TMB} \geq 10 \mathrm{mut} / \mathrm{Mb}$ was associated with overall survival determined from the time of diagnosis in patients stratified by race and treatment type, noting that statistical power to detect associations may be limited for minorities due to smaller sample sizes.

After adjusting for sex, age, and cancer type, TMBhigh cancers in the non-ICI cohort were significantly associated with worse overall survival among white (HR 1.33; $95 \%$ CI 1.11 to 1.59 ) and Asian (HR 2.29; 95\% CI 1.22 to 4.29 ) patients (figure 2A). TMB-high cancers also demonstrated a trend toward worse overall survival among Black patients (HR 1.75; 95\% CI 0.90 to 3.40). In contrast, TMB-high cancers in the ICI cohort were associated with improved overall survival among White patients (HR 0.62; 95\% CI 0.52 to 0.74 ) and had no significant association with survival among black (HR 0.99; 95\% CI 0.49 to 1.99 ) and Asian patients ((HR 0.55; 95\% CI 0.20 to 1.52) (figure 2A). These results indicate that the association between TMB-high cancers and overall survival is dependent on treatment context regardless of race.

\section{TMB cutoffs less than $10 \mathrm{mut} / \mathrm{Mb}$ may be predictive of ICI benefit across racial groups}

To assess the predictive value of TMB cutoffs among patients across different racial groups, we assessed the objective response rate (including partial and complete responses) to ICIs among 1594 patients in the ICIcohort with available response data after stratifying patients by race. By varying cutoffs to define TMBhigh cancers, we found that a TMB cut-off of at least 6 mut/Mb was still significantly associated with a higher probability of objective response in white and black patients (figure 2B). In Asian patients, a trend association towards increased objective responses was observed beginning with a TMB cut-off of 8 mut/Mb (figure 2B). Cox regression analyses adjusting for age, sex, and cancer type showed that progression-free survival, defined as the time from the initiation of an ICI to disease progression or death, was also greater in TMB-high cancers when using cut-off thresholds of at least 8 mut/Mb across all races (online supplemental figure 3A). Cox regression analyses of overall survival did not demonstrate an association between any cut-off threshold and survival for black or Asian patients, but the much smaller sample sizes of minority patients in these analyses does not preclude the possibility of a survival benefit (online supplemental figure 3B). Collectively, these results suggest that a range of TMB thresholds have similar predictive value of ICI benefit across race.

\section{DISCUSSION}

Our analyses demonstrate that the distribution of TMB values in tumors may differ among patients of different racial groups, and that the predictive value of high TMB is similar in both ICI and non-ICI contexts. Because of these differences, the use of a single, tissue-agnostic definition of high TMB to define eligibility for anti-PD-1 therapy will not necessarily be a mechanism to alleviate disparities in access to these treatments. To achieve the goal of reducing disparate eligibility for anti-PD-1 therapy under current FDA approvals, the numerical definition of high TMB may need to be set at a lower number. However, the clinical and economic value of doing so remains to be clarified, as it remains to be tested whether such a change would improve survival outcomes or cost-effectiveness. While this study leverages among the largest genomic datasets with available race data and ICI outcomes, the limited sample size of minority patients precludes precise definitions of optimal TMB cutoffs for individual cancer types. Nonetheless, this work underscores a clear need to understand mechanisms of TMB variation across race, and the importance of including minorities within ICI studies to clarify the differential impact of predictive biomarkers.

The biological basis for the differing prevalence of TMB-high cancers between racial groups needs to be clarified, but genomic differences in cancers are known to exist among different racial populations. While we adjusted for age and sex, other clinical factors likely to contribute to TMB such as smoking history and it remains to be investigated whether other patient characteristics may explain a lower frequency of TMB-high cancers in minorities.

There are several limitations to this analysis including the fact that neither the TCGA or MSK-IMPACT cohorts were designed to be representative of the US population and the lack of sufficient data to assess other minority races. Self-reported data on race as used in this study is 
A

\begin{tabular}{|c|c|c|c|c|}
\hline & & Overall survival hazard ratio & $\mathrm{N}$ & $\operatorname{HR}(95 \% \mathrm{Cl})$ \\
\hline \multirow{3}{*}{$\begin{array}{l}\text { Non-ICl } \\
\text { cohort }\end{array}$} & White & $1+1$ & 6396 & $1.33(1.11-1.59)$ \\
\hline & Black & 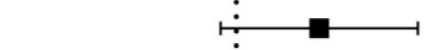 & 553 & $1.75(0.90-3.40)$ \\
\hline & Asian & $\longmapsto$ & 651 & $2.29(1.22-4.29)$ \\
\hline \multirow{3}{*}{$\begin{array}{c}\text { ICI } \\
\text { cohort }\end{array}$} & White & $\mapsto-1$ & 1604 & $0.62(0.52-0.74)$ \\
\hline & Black & -1 & 128 & $0.99(0.49-1.99)$ \\
\hline & Asian & $\longrightarrow$ & 135 & $0.55(0.20-1.52)$ \\
\hline
\end{tabular}

B White $(\mathrm{N}=1371)$

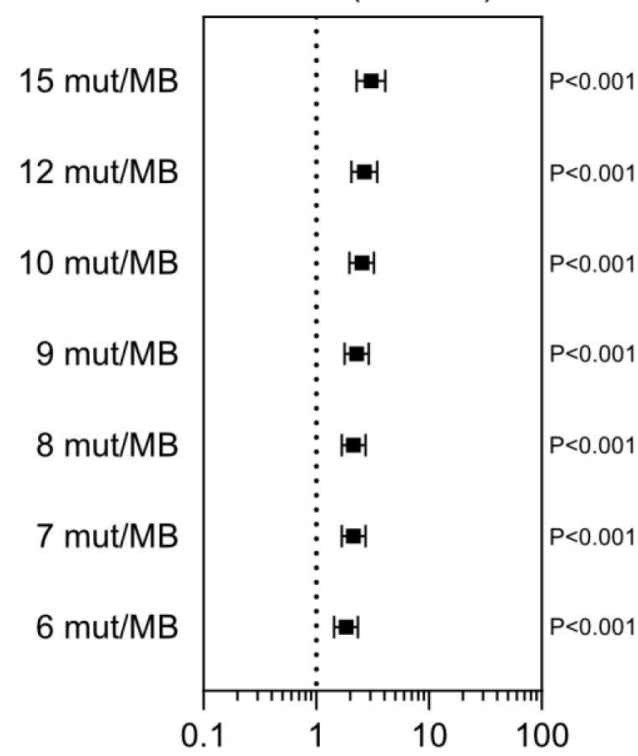

Black ( $\mathrm{N}=111)$

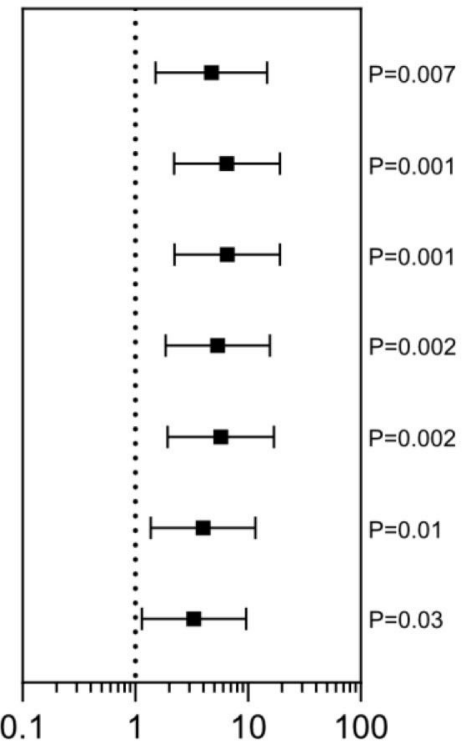

Asian $(\mathrm{N}=112)$

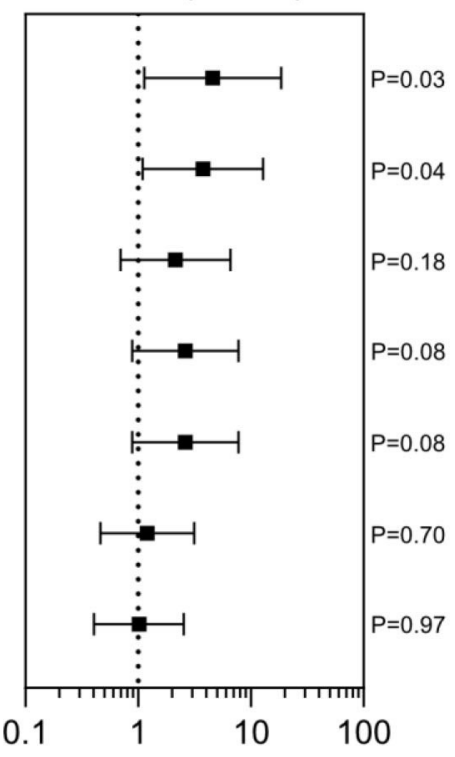

Odds ratio of objective response

Figure 2 Association between tumor mutation burden and overall survival across race. (A) Multivariable Cox regression analysis of the non-immune checkpoint inhibitor $(\mathrm{ICl})$ cohort and $\mathrm{ICl}$ cohort after stratifying patients by race was performed with overall survival as the endpoint. HRs adjusted for age, sex, and cancer type indicate the association between TMB-high or TMB-low status and overall survival. (B), Logistic regression was performed in the ICl cohort after stratifying patients by race using different cutoffs to define TMB-high cancers. ORs indicate the odds of an objective response in TMB-high vs TMB-low cancers. The error bars represent the $95 \%$ Cls. All statistical tests were two sided.

the preferred method for collecting race data in most biomedical studies, but is associated with several limitations including a limited relevance to human genetic variation. Estimating race through genetic ancestry is possible with high-density genotyping, but this approach is not applicable to most patients examined in this study and may be less relevant to characterizing healthcare disparities. Nonetheless, analysis of patients categorized by genetic ancestry in the TCGA cohort showed a consistent enrichment of TMB-low cancers in patients of African and Asian descent.

There is conflicting evidence on the use of TMB as a predictive marker across multiple cancer types, and whether TMB cutoffs should be different for specific cancer types remains to be determined. Given the absence of reliable or validated predictive cancer-specific TMB cutoffs, it remains to be determined how alternative definitions of TMB-high cancers may impact ICI eligibility across racial groups. It should be also noted that the definition of high TMB in the current FDA approval is based on a commercial gene panel companion diagnostic, and whether the current cut-off of 10 mut/Mb can be reliably used in other assays remains to be clarified. In addition, methods used to estimate TMB remain to be standardized, and efforts to harmonize assays, sequencing technologies, and computational algorithms are underway. ${ }^{10}$

Given the limited number of minority patients with available treatment data across different cancer types, our conclusions regarding the predictive value of TMB across racial groups requires further study. Limited sample sizes 
of minority patients treated with ICIs likely contributed to the lack of a detectable association between overall survival after ICI treatment and TMB-high cancers in Black and Asian patients. Nonetheless, objective response rates and progression-free survival were demonstrated in our study to be greater among TMB-high cancers even when using lower TMB thresholds than the current FDA approval. These results are meaningful given that the FDA approval for the use of a TMB cut-off of 10 mut/Mb was only based on objective response rates.

This work highlights an under-recognized challenge of expanding precision oncology particularly when minorities are under-represented in trials and sequencing datasets used to guide biomarker and treatment approvals. Our work also indicates a need for further race-conscious research particularly in the development of predictive biomarkers and evaluation of cancer treatments. Given a dearth of knowledge on the effect of race on immunotherapy outcomes, additional studies are needed to identify more rationale or equitable strategies to expand immunotherapy eligibility.

\section{Author affiliations}

${ }^{1}$ Division of Hematology and Oncology, Department of Internal Medicine, University of Texas Southwestern Medical Center, Dallas, Texas, USA

${ }^{2}$ Harold C. Simmons Comprehensive Cancer Center, University of Texas

Southwestern Medical Center, Dallas, Texas, USA

${ }^{3}$ Division of Digestive and Liver Diseases, Department of Internal Medicine, University of Texas Southwestern Medical Center, Dallas, Texas, USA

${ }^{4}$ Department of Surgery, Memorial Sloan Kettering Cancer Center, New York, New York, USA

${ }^{5}$ Department of Population and Data Sciences, University of Texas Southwestern Medical Center, Dallas, Texas, USA

\begin{abstract}
Acknowledgements This work was supported by a Cancer Prevention and Research Institute of Texas career award RP200549 (to DH) and an NIH grant R01DE027738 (to LGTM). Both authors declare no conflicts of interest. LGTM is an inventor on a provisional patent application $(62 / 569,053)$ filed by MSKCC, relating to the use of TMB in cancer immunotherapy. All other authors declare no competing interests. Both authors wrote the paper and approve the final version of the paper. The results reported here are in part based on data generated by the TCGA Research Network: https://www.cancer.gov/tcga.
\end{abstract}

Contributors $\mathrm{DH}$ conceived the study. DH, ME, and CA performed data analyses. CV and LGTM contributed data. All authors wrote the paper. DH is the guarantor of the study.

Funding Cancer Prevention and Research Institute of Texas (RP200549), National Institute of Dental and Craniofacial Research (R01DE027738).

Competing interests None declared.
Patient consent for publication Not applicable.

Provenance and peer review Not commissioned; externally peer reviewed.

Data availability statement Genomic and demographic data analyzed in the current study in the TCGA or MSK-IMPACT datasets are available at https://portal. gdc.cancer.gov and cbioportal.org.

Supplemental material This content has been supplied by the author(s). It has not been vetted by BMJ Publishing Group Limited (BMJ) and may not have been peer-reviewed. Any opinions or recommendations discussed are solely those of the author(s) and are not endorsed by BMJ. BMJ disclaims all liability and responsibility arising from any reliance placed on the content. Where the content includes any translated material, BMJ does not warrant the accuracy and reliability of the translations (including but not limited to local regulations, clinical guidelines, terminology, drug names and drug dosages), and is not responsible for any error and/or omissions arising from translation and adaptation or otherwise.

Open access This is an open access article distributed in accordance with the Creative Commons Attribution Non Commercial (CC BY-NC 4.0) license, which permits others to distribute, remix, adapt, build upon this work non-commercially, and license their derivative works on different terms, provided the original work is properly cited, appropriate credit is given, any changes made indicated, and the use is non-commercial. See http://creativecommons.org/licenses/by-nc/4.0/.

\section{REFERENCES}

1 Rizvi NA, Hellmann MD, Snyder A, et al. Cancer immunology. Mutational landscape determines sensitivity to PD-1 blockade in non-small cell lung cancer. Science 2015;348:124-8.

2 Snyder A, Makarov V, Merghoub T, et al. Genetic basis for clinical response to CTLA-4 blockade in melanoma. $N$ Engl $\mathrm{J}$ Med 2014;371:2189-99.

3 Yarchoan M, Hopkins A, Jaffee EM. Tumor mutational burden and response rate to PD-1 inhibition. N Engl J Med 2017;377:2500-1.

4 Chan TA, Yarchoan M, Jaffee E, et al. Development of tumor mutation burden as an immunotherapy biomarker: utility for the oncology clinic. Ann Oncol 2019;30:44-56.

5 Marabelle A, Le DT, Ascierto PA, et al. Efficacy of pembrolizumab in patients with Noncolorectal high microsatellite instability/mismatch repair-deficient cancer: results from the phase II KEYNOTE-158 study. J Clin Oncol 2020;38:1-10.

6 Prasad V, Addeo A. The FDA approval of pembrolizumab for patients with TMB >10 mut/Mb: was it a wise decision? No. Ann Oncol 2020;31:1112-4.

7 Subbiah V, Solit DB, Chan TA, et al. The FDA approval of pembrolizumab for adult and pediatric patients with tumor mutational burden $(T M B) \geq 10$ : a decision centered on empowering patients and their physicians. Ann Oncol 2020;31:1115-8.

8 Carrot-Zhang J, Chambwe N, Damrauer JS, et al. Comprehensive analysis of genetic ancestry and its molecular correlates in cancer. Cancer Cell 2020;37:639-54.

9 Valero C, Lee M, Hoen D, et al. The association between tumor mutational burden and prognosis is dependent on treatment context. Nat Genet 2021;53:11-15.

10 Merino DM, McShane LM, Fabrizio D, et al. Establishing guidelines to harmonize tumor mutational burden (TMB): in silico assessment of variation in TMB quantification across diagnostic platforms: phase I of the friends of cancer research TMB harmonization project. $\mathrm{J}$ Immunother Cancer 2020;8:e000147. 